\title{
Photoinduced Enhancement of Excitonic Order
}

\author{
Yuta Murakami, ${ }^{1}$ Denis Golež, ${ }^{1}$ Martin Eckstein, ${ }^{2}$ and Philipp Werner ${ }^{1}$ \\ ${ }^{1}$ Department of Physics, University of Fribourg, 1700 Fribourg, Switzerland \\ ${ }^{2}$ Department of Physics, University of Erlangen-Nürnberg, 91058 Erlangen, Germany
}

(Received 10 May 2017; published 15 December 2017)

\begin{abstract}
We study the dynamics of excitonic insulators coupled to phonons using the time-dependent mean-field theory. Without phonon couplings, the linear response is given by the damped amplitude oscillations of the order parameter with a frequency equal to the minimum band gap. A phonon coupling to the interband transfer integral induces two types of long-lived collective oscillations of the amplitude, one originating from the phonon dynamics and the other from the phase mode, which becomes massive. We show that, even for small phonon coupling, a photoinduced enhancement of the exciton condensation and the gap can be realized. Using the Anderson pseudospin picture, we argue that the origin of the enhancement is a cooperative effect of the massive phase mode and the Hartree shift induced by the photoexcitation. We also discuss how the enhancement of the order and the collective modes can be observed with time-resolved photoemission spectroscopy.
\end{abstract}

DOI: 10.1103/PhysRevLett.119.247601

Introduction.-Nonequilibrium dynamics can provide new insights into properties of materials and new ways to control ordered states. In this context, superconducting (SC) phases have been studied extensively. Both a light-induced enhancement of SC [1-3] and the observation of the amplitude Higgs mode [4-6] have been reported. Recently, also a related family of ordered states, i.e., excitonic insulators (EIs), has attracted interest [7-12]. An EI state is formed by the macroscopic condensation of electron-hole pairs $[13,14]$, and its theoretical description is analogous to the BCS or Bose-Einstein condensate (BEC) theory for SC. Although the idea of exciton condensation was proposed already in the 1960s [13-15], the interest in this topic has been renewed recently by the study of some candidate materials such as $1 T-\mathrm{TiSe}_{2}$ and $\mathrm{Ta}_{2} \mathrm{NiSe}_{5}$ (TNS) [16-21]. Their analogy to SC makes the EI an interesting system for nonequilibrium studies. In particular, for TNS, time- and angle-resolved photoemission spectroscopy (trARPES) experiments showed that the direct band gap can be either decreased or increased depending on the pump fluence [11], which was interpreted in terms of a photoinduced enhancement or suppression of excitonic order. A more recent report of the amplitude mode [12] provides further confirmation of an EI state in TNS.

So far, the theoretical works on EIs have mainly focused on the equilibrium properties such as the BEC-BCS crossover [22,23], the coupling of the EI to phonons [24-26], linear susceptibilities [27-29], and the effect of strong interactions and new emergent phases [30-32]. In contrast, the nonequilibrium investigation of EIs has just begun [10]. In this work, using TNS as a model system, we clarify two basic effects of the electron-phonon (e-ph) coupling on the dynamics of EIs: (i) the effect on collective modes and (ii) the impact on the excitonic order after photoexcitation. In particular, we show that with phonons photoexcitation can result in an enhancement of the order.

Formalism.-In this Letter, we focus on a two-band model of spinless electrons coupled to phonons:

$$
\begin{aligned}
\hat{H}(t)= & \sum_{k, \alpha=0,1}\left(\epsilon_{k, \alpha}+\Delta_{\alpha}\right) \hat{c}_{k, \alpha}^{\dagger} \hat{c}_{k, \alpha} \\
& +E(t) \sum_{k}\left(\hat{c}_{k, 1}^{\dagger} \hat{c}_{k, 0}+\hat{c}_{k, 0}^{\dagger} \hat{c}_{k, 1}\right)+U \sum_{i} \hat{n}_{i, 0} \hat{n}_{i, 1} \\
& +g \sum_{i}\left(\hat{b}_{i}^{\dagger}+\hat{b}_{i}\right)\left(\hat{c}_{i, 1}^{\dagger} \hat{c}_{i, 0}+\hat{c}_{i, 0}^{\dagger} \hat{c}_{i, 1}\right)+\omega_{0} \sum_{i} \hat{b}_{i}^{\dagger} \hat{b}_{i} .
\end{aligned}
$$

In order to mimic the quasi-one-dimensionality and direct band gap in TNS, we consider a one-dimensional configuration with $N$ sites $(N \rightarrow \infty) . \hat{c}_{i, \alpha}^{\dagger}$ is the creation operator of an electron at site $i$ in band $\alpha(\alpha=0,1$ for the conduction and valence bands, respectively). $k$ indicates the momentum, $c_{k, \alpha}^{\dagger}=(1 / \sqrt{N}) \sum_{j} e^{i k \cdot j} c_{j, \alpha}^{\dagger}$, and $\hat{n}_{i, \alpha}=\hat{c}_{i, \alpha}^{\dagger} \hat{c}_{i, \alpha} \cdot \Delta_{\alpha}$ is the crystal field, and we choose $\epsilon_{k, \alpha}=-2 J(-1)^{\alpha} \cos (k)$. $E(t)$ denotes the external laser field, and we assume that the laser couples to the system through dipolar transitions. $U$ is the interaction between bands and the driving force for the excitonic pairing. We denote the phonon creation operator at site $i$ by $\hat{b}_{i}^{\dagger}$, the $e$-ph coupling by $g$, and the phonon frequency by $\omega_{0}$, and define the effective $e$-ph interaction by $\lambda \equiv 2 g^{2} / \omega_{0}$. This phonon is associated with a lattice distortion in TNS, which hybridizes the electronic bands [24]. The hopping parameter $J$ is our unit of energy.

For $g=0$ with $\Delta_{0} \neq \Delta_{1}$, the system has a U(1) symmetry (corresponding to the conservation of the particle number in each band), which is broken in the EI. In the $e$-ph coupled system $(g \neq 0)$, this $\mathrm{U}(1)$ symmetry is reduced to a 
$Z_{2}$ symmetry, and we will see that this has profound consequences for the collective modes and the nonequilibrium dynamics.

The dynamics of the system is studied within the time-dependent mean-field theory at $T=0$ [33]. We define the order parameter of the excitonic condensate as $\phi(t) \equiv\left\langle\hat{c}_{i, 0}^{\dagger}(t) \hat{c}_{i, 1}(t)\right\rangle$, which we take real in equilibrium, the difference in occupancy between the conduction and valence bands $\Delta n(t) \equiv\left\langle\hat{n}_{i, 0}(t)\right\rangle-\left\langle\hat{n}_{i, 1}(t)\right\rangle$, and the phonon displacements $X(t) \equiv\left\langle\hat{b}_{i}^{\dagger}(t)+\hat{b}_{i}(t)\right\rangle$. The choice of $i$ does not matter because of the homogeneous excitation. The mean-field time evolution is self-consistently determined through $\phi(t), \Delta n(t)$, and $X(t)$. This can be simply expressed in a pseudospin representation, $\hat{S}_{k}^{\gamma} \equiv \hat{\Psi}_{k}^{\dagger} \frac{1}{2} \hat{\sigma}_{\gamma} \hat{\Psi}_{k}$ with the spinor $\hat{\Psi}_{k}^{\dagger} \equiv\left[\hat{c}_{k, 0}^{\dagger}, \hat{c}_{k, 1}^{\dagger}\right]$, in analogy to the Anderson pseudospin representation [34]. Here $\hat{\sigma}_{\gamma}$ for $\gamma=x, y, z$ denotes the Pauli matrix, and $\gamma=0$ is the identity matrix. The spin commutation relation is fulfilled: $\left[\hat{S}^{\alpha}, \hat{S}^{\beta}\right]=$ $i \epsilon_{\alpha, \beta, \gamma} \hat{S}^{\gamma}$ (except for $\hat{S}^{0}$, which commutes with all other operators). With these operators, $\Delta n(t)=(2 / N) \sum_{k}\left\langle\hat{S}_{k}^{z}(t)\right\rangle$, the total number of particles per site is $\left\langle\hat{n}_{0}(t)\right\rangle+\left\langle\hat{n}_{1}(t)\right\rangle=$ $(2 / N) \sum_{k}\left\langle\hat{S}_{k}^{0}(t)\right\rangle$, and the order parameter is $\phi(t)=$ $(1 / N) \sum_{k}\left\langle\hat{S}_{k}^{x}(t)+i \hat{S}_{k}^{y}(t)\right\rangle$.

In the mean-field description, the time evolution of electrons is expressed using the pseudospin expectation values as

$$
\partial_{t} \mathbf{S}_{k}(t)=\mathbf{B}_{k}(t) \times \mathbf{S}_{k}(t),
$$

with a pseudomagnetic field $\mathbf{B}_{k}(t)$ :

$$
\begin{aligned}
& B_{k}^{x}(t)=2[E(t)+g X(t)-U \operatorname{Re} \phi(t)], \\
& B_{k}^{y}(t)=-2 U \operatorname{Im} \phi(t), \\
& B_{k}^{z}(t)=2 \epsilon_{k}^{z}-U \Delta n(t),
\end{aligned}
$$

where $2 \epsilon_{k}^{z} \equiv\left(\epsilon_{k, 0}-\epsilon_{k, 1}\right)+\left(\Delta_{0}-\Delta_{1}\right)$. As for the phonons, $\partial_{t}^{2} X(t)=-\omega_{0}^{2} X(t)-2 g \omega_{0}\left[\phi(t)+\phi(t)^{*}\right]$ with $\left.\partial_{t} X(t)\right|_{t=0}=0$. Equations (3) imply that in the normal state [with $E(t)=0$ ] the pseudomagnetic field and pseudospins are along the $z$ axis, while in the EI state there are additional $x$ or $y$ components. In particular, the phonon contributes to the $x$ component.

Results.-We choose the reference parameters such that the model with $\lambda=0$ reproduces the ARPES spectra of TNS [11], namely, $\Delta_{0}=-0.55, \Delta_{1}=-2.45, \lambda=0$, and $U=3$, which is half filled and in the BEC regime [22,35]. The minimum gap between the quasiparticle bands $\Delta_{\mathrm{EI}}$ is located at $k=0$ in equilibrium. In the calculations with $\lambda>0$, we adjust $\Delta_{\alpha}$ and $U$ such that in equilibrium the electronic properties (i.e., $\phi, \Delta n$, and the quasiparticle

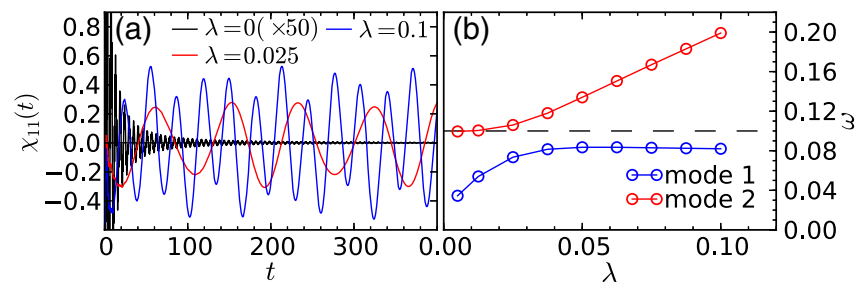

FIG. 1. (a) Susceptibility $\chi_{11}^{R}(t)$ for different couplings $\lambda$. (b) $\lambda$ dependence of the frequencies of the collective modes at $q=0$ extracted from the peak positions in $-\operatorname{Im} \chi_{11}^{R}(\omega)$. The dashed black line indicates $\omega_{0}$.

dispersion) are the same as for $\lambda=0$. In the following, we fix $\omega_{0}=0.1$.

We start with the linear response regime and investigate the collective modes at $q=0$ by analyzing the linear susceptibility $\chi_{11}^{R}(t) \equiv-i \theta(t) N\left\langle\left[\hat{\rho}_{1}(t), \hat{\rho}_{1}(0)\right]\right\rangle$, with $\hat{\rho}_{1}=$ $(1 / N) \sum_{i}\left(\hat{c}_{i, 1}^{\dagger} \hat{c}_{i, 0}+\hat{c}_{i, 0}^{\dagger} \hat{c}_{i, 1}\right)$ [36]. Since the initial $\phi$ is real, $\chi_{11}^{R}$ captures the dynamics of the amplitude of the order parameter $\phi(t)$. Note that $\chi_{11}^{R}$ corresponds to the dynamical pair susceptibility in SCs, which has been used to study the amplitude Higgs mode [37-39].

In Fig. 1(a), we show $\chi_{11}^{R}(t)$ for different phonon couplings. Without phonons, $\chi_{11}^{R}(t)$ oscillates with the same frequency as the minimum gap $\Delta_{\mathrm{EI}}=1.15$, and its amplitude decays as $1 / t^{1.5}$ [see Fig. 1(a)], which is consistent with the previous prediction for strong-coupling SC [40]. (In contrast, the amplitude oscillations in the BCS regime decay as $1 / t^{0.5}$ [33], which is also consistent with the corresponding predictions for SC [41-44]. Therefore, the existence of prominent amplitude oscillations with the frequency of the gap and a decay $\sim 1 / t^{0.5}$ may be used to distinguish the BCS from the BEC nature of the system [21].)

For $\lambda=0$, the Hamiltonian has a U(1) symmetry, and in the EI phase a massless phase mode emerges (the Goldstone mode). The $e$-ph coupling breaks the $\mathrm{U}(1)$ symmetry, and this massless mode becomes massive $[25,45]$, which leads to additional (undamped) oscillations with two different frequencies in $\chi_{11}^{R}(t)$; see Fig. 1(a) and Ref. [33]. In Fig. 1(b), we show the dependence of these frequencies on $\lambda$ [46]. The mode whose energy grows from $\omega=0$ corresponds to the massive phase mode. (It shows a strong signal in the susceptibility for the phase direction of the order parameter [33].) The mixing between the amplitude and phase oscillations distinguishes EIs from BCS SCs. The other mode, whose frequency increases from $\omega=\omega_{0}$, can be regarded as the phonon mode.

Next, we consider the excitation with a laser pulse and discuss how these different properties of the collective modes show up out of equilibrium, and under which conditions the order can be enhanced. We prepare the equilibrium state at $T=0$ at $t=0$ and choose $E(t)=$ $E_{0} \sin (\Omega t) \exp \left[-\left(t-t_{p}\right)^{2} /\left(2 \sigma_{p}^{2}\right)\right]$ with $\Omega=6$ and $\sigma_{p}=3$, which corresponds to a $1.56 \mathrm{eV}$ frequency multicycle laser 
pulse. With this choice of parameters, the electrons are directly excited from the lower part of the valence band into the upper part of the conduction band.

In Fig. 2, we show the time evolution of the gap at $k=0$ $[\Delta(k=0, t)]$, the absolute value of the order parameter $(|\phi|)$, and the phonon displacement $(X)$ after the pulse for various conditions [47]. When the system is coupled to phonons, we find that $\Delta(k=0, t),|\phi|$, and $|X|$ are enhanced; see Figs. 2(a)-2(c). These quantities oscillate with two characteristic frequencies corresponding to the massive phase mode and the phonon mode, as discussed in the linear response regime. With increasing field strength, the enhancement of these quantities becomes more prominent, and the frequencies of the oscillations are slightly increased. On the other hand, the system without phonons shows a suppression of the gap and the order parameter; see Figs. 2(a) and 2(b). The dependence of the time average of $|\phi|$ on the $e$-ph coupling [Fig. 2(d)] shows that a weak coupling already leads to the enhancement of the order. At a critical coupling $\lambda_{c}$, the system exhibits a dynamical phase transition (discussed below).

For the enhancement of the order, the Hartree shift is essential. After photoexcitation, the difference in the occupation, $-\Delta n$, is reduced, which leads to a substantial Hartree shift (i.e., a decrease of $B_{k}^{z}$ ), and the bare band gap
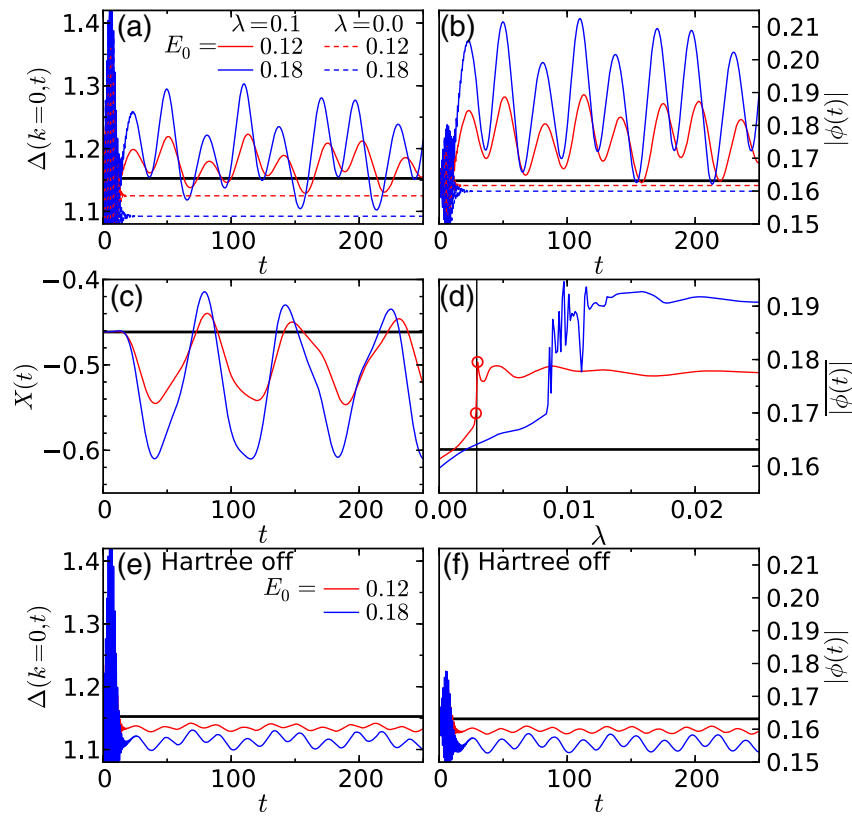

FIG. 2. (a)-(c) Time evolution of the gap at $k=0, \Delta(k=0, t)$, the excitonic order parameter $|\phi|$, and the phonon displacement $X$ for various field strengths and $e$-ph couplings. (d) $\lambda$ dependence of $|\phi|$ averaged over $t \in[0,400]$, for various field strengths. The vertical line indicates the critical value $\lambda_{c}$ at $E_{0}=0.12$. (e),(f) Time evolution of $\Delta(k=0, t)$ and $|\phi|$ for $\lambda=0.1$ evaluated by freezing the Hartree shift. We have used $t_{p}=6.0$. The horizontal black line in each panel shows the corresponding equilibrium values. becomes smaller. If the Hartree shift is artificially suppressed ( $B^{z}$ fixed) in the mean-field dynamics, one finds a suppression of $\Delta(k=0, t)$ and $|\phi|$; see Figs. 2(e) and 2(f). In equilibrium, the smaller distance between bands leads to enhanced excitonic condensation. This argument, however, assumes thermal distribution functions and cannot be used to explain the transient state. The pseudospin picture, on the other hand, suggests an interesting scenario for how the change of $B^{z}$ enhances the order: If $B^{x}$ and $B^{y}$ would remain static after the sudden decrease of $B^{z}$ due to the Hartree shift, the pseudomagnetic field would tilt more to the $x$ direction compared to the equilibrium case. This would lead to a spin precession around the tilted magnetic field, which yields a larger projection on the $x-z$ plane, $\left|S_{k}^{x}+i S_{k}^{y}\right|$, and therefore an enhancement of the order parameter.

In reality, however, the evolution of the pseudomagnetic field $B^{x}$ and $B^{y}$ and the pseudospin must be determined self-consistently. In Fig. 3, we show how they evolve with and without phonons. We take $k=0$ as a representative,
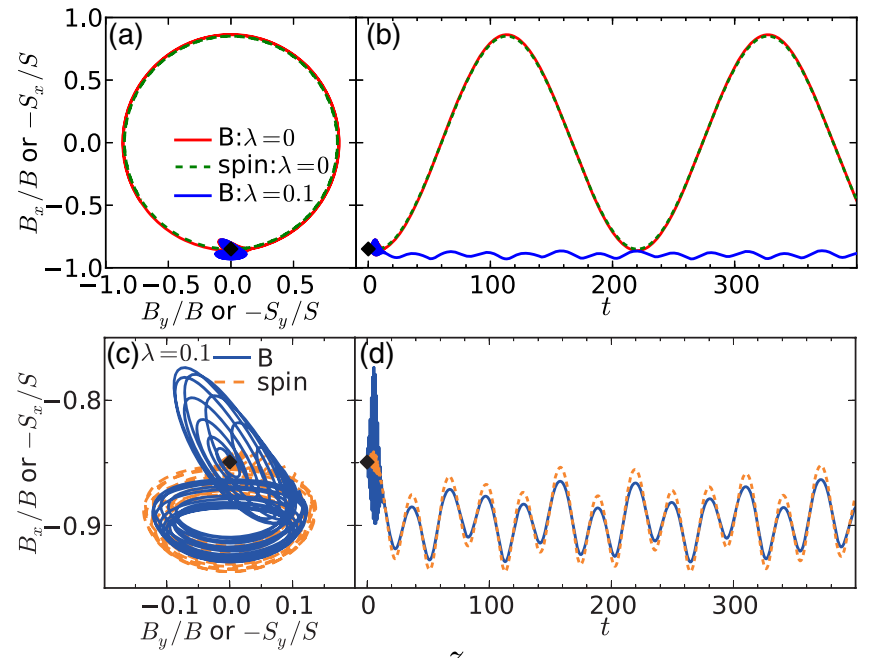

(e)

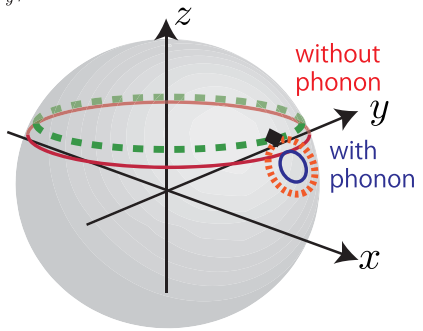

FIG. 3. (a) Trajectory of the normalized pseudomagnetic field $\left(B^{x} / B, B^{y} / B\right)$ and the normalized pseudospin $\left(-S^{x} / S,-S^{y} / S\right)$ at $k=0$ with and without phonons. (b) The time evolution of $B^{x} / B$ and $-S^{x} / S$ at $k=0$ with and without phonons. (c) The magnified trajectory of $\left(B^{x} / B, B^{y} / B\right)$ and $\left(-S^{x} / S,-S^{y} / S\right)$ and (d) the time evolution of $B^{x} / B$ and $-S^{x} / S$ at $k=0$ for $\lambda=0.1$. The parameters are $t_{p}=6.0$ and $E_{0}=0.12$. (e) Schematic picture of the trajectory of $\mathbf{B} / B$ (solid lines) and $-\mathbf{S} / S$ (dashed lines) with and without phonons. The equilibrium positions of $\mathbf{B} / B$ and $-\mathbf{S} / S$ are indicated by a black diamond in each panel. 
since the region around $k=0$ has the largest contribution to the excitonic condensation. For $\lambda=0$, the phase mode is massless; hence, after the excitation the magnetic field rotates around the $z$ axis following the minimum of the free energy. The spin follows the magnetic field by keeping the relative angle. Hence, the spin cannot precess around the magnetic field and cannot realize the enhancement of $\mid S_{k}^{x}+$ $i S_{k}^{y} \mid$ from a rotation around a fixed and tilted field as discussed above; see Figs. 3(a), 3(b), and 3(e). On the other hand, for $\lambda>0$ the phase mode is massive, and therefore the magnetic field is almost confined to the $x-z$ plane; see Figs. 3(a) and 3(c). This means that even in the selfconsistent case, when the EI is coupled to phonons, the result is close to the naive static picture (fixed $B^{x}$ and $B^{y}$ ), where the enhancement of the order is explained as a precession around the tilted magnetic field in the $x$ direction. Even though the increase of the projection to the $x-y$ plane, $\left|S_{k}^{x}+i S_{k}^{y}\right|$, after photoexcitation tends to enhance the order, the phases of $S_{k}^{x}+i S_{k}^{y}$ for different momenta $k$ can lead to destructive interference and a decrease of the order. However, it turns out that the pseudospin at each $k$ roughly follows the magnetic field [see Fig. 3(d)] so that this effect is small. We have confirmed that this mechanism, which is based on the massive phase mode, is robust against the frequency of the phonons and the number of phonon branches [33].

With increasing field strength, the amplitude of the oscillations of the magnetic field around the $x-z$ plane becomes large. At some critical strength (which depends on $\lambda$ ), it can overcome the potential barrier of the free energy along the phase direction, which is associated with the massive phase mode, and starts to rotate around the $z$ axis [33]. This can be regarded as a dynamical phase transition [cf. critical $\lambda_{c}$ in Fig. 2(d)] [48-55], which is of firstorder type.

Finally, we discuss how the enhancement of the excitonic order and gap can be observed in experiments. In Figs. 4(a) and 4(b), we show the trARPES spectrum evaluated as $[56,57] A_{k}^{R}\left(\omega ; t_{\mathrm{pr}}\right)=-(1 / \pi) \operatorname{Im} \iint d t d t^{\prime} s\left(t-t_{\mathrm{pr}}\right) s\left(t^{\prime}-t_{\mathrm{pr}}\right) \times$ $e^{i \omega\left(t-t^{\prime}\right)}\left[G_{k, 00}^{R}\left(t, t^{\prime}\right)+G_{k, 11}^{R}\left(t, t^{\prime}\right)\right] / 2$ before and after the laser pump. Here $G_{k, \alpha \beta}^{R}\left(t, t^{\prime}\right)=-i \theta\left(t-t^{\prime}\right)\left\langle\left[\hat{c}_{k, \alpha}(t), \hat{c}_{k, \beta}^{\dagger}\left(t^{\prime}\right)\right]_{+}\right\rangle$is the retarded part of the Green's function, $s(t)=$ $\left[1 /\left(\sqrt{2 \pi} \sigma_{\mathrm{pr}}\right)\right] \exp \left[-t^{2} /\left(2 \sigma_{\mathrm{pr}}^{2}\right)\right], t_{\mathrm{pr}}$ is the probe time, and $\sigma_{\mathrm{pr}}$ is the width of the probe pulse. Before the pump, the peak in the spectrum follows the quasiparticle dispersion from the mean-field theory in equilibrium. After the pump, the weight of the spectrum shifts away from the Fermi level around $k=0$ (the $\Gamma$ point), while away from $k=0$ it shifts closer to the Fermi level. In Fig. 4(c), we show the time evolution of the difference between the equilibrium and nonequilibrium band distance at each $k\left[\delta \Delta_{\mathrm{ARPES}}\left(k, t_{\mathrm{pr}}\right)\right]$, which is determined from the difference between the peaks in $A_{k}^{R}\left(\omega ; t_{\mathrm{pr}}\right)$. The shift in the band distance oscillates with the frequencies observed in the analysis of $\chi_{11}$ [the orange line in Fig. 4(c)],
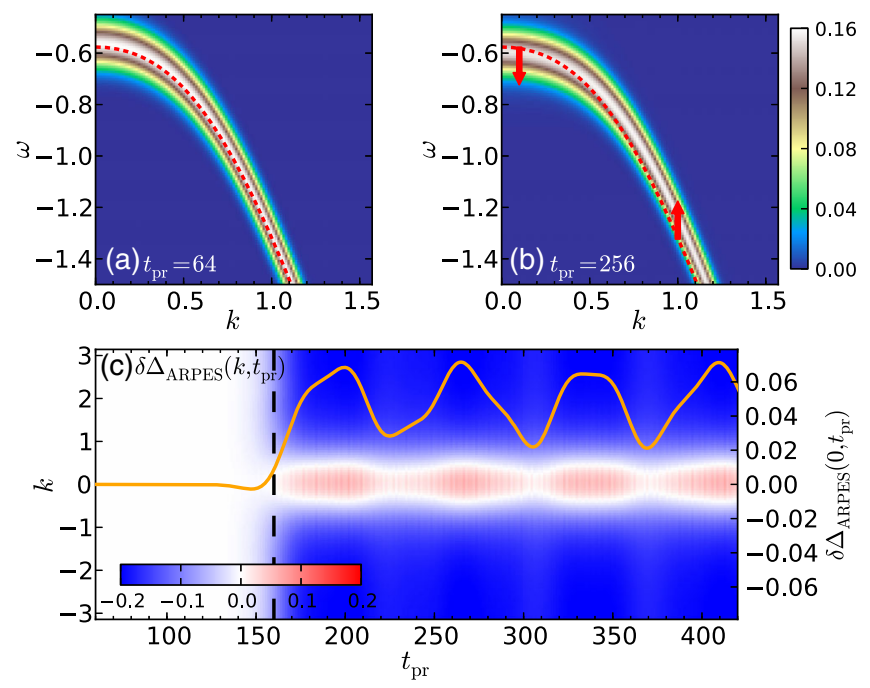

FIG. 4. (a),(b) trARPES spectra $\left[A_{k}^{R}\left(\omega ; t_{\mathrm{pr}}\right)\right]$ before (a) and after (b) the pump. Red dashed lines indicate the equilibrium quasiparticle dispersion from the mean-field theory. (c) Time evolution of the difference between the equilibrium and nonequilibrium gap size at each momentum $k\left[\delta \Delta_{\mathrm{ARPES}}\left(k, t_{\mathrm{pr}}\right)\right]$. The orange solid line is $\delta \Delta_{\text {ARPES }}\left(0, t_{\mathrm{pr}}\right)$. The parameters are $\lambda=0.1, E_{0}=0.18$, $t_{p}=160.0$, and $\sigma_{\mathrm{pr}}=12.0$. The black dashed line indicates the center of the pump pulse.

which demonstrates that the collective modes can be observed with trARPES.

Conclusions.-We have revealed that the $e$-ph coupling, which is associated with the structural transition in $\mathrm{Ta}_{2} \mathrm{NiSe}_{5}$, has a large and qualitative effect on the dynamics of the excitonic insulator. In particular, we demonstrated a novel mechanism for photoenhanced excitonic order based on a cooperative effect between the $e$-ph coupling, the massive phase mode, and the Hartree shift. Combining photoexcitation and a reduction of the symmetry of the Hamiltonian may provide a new strategy to enhance analogous orders such as SC [58].

The proposed mechanism for photoenhanced excitonic order is an alternative to the one discussed in Ref. [11], where the enhancement was attributed to nonthermal distribution functions with additional Hartree shifts from photodoping to higher conduction bands. Both mechanisms are consistent with the experimental results reported in Ref. [11], which show a gap enhancement around the $\Gamma$ point and a reduction away from it. We believe that they could be combined; e.g., at short times the drastic effects of the $e$-ph coupling and the additional Hartree shifts cooperate, while the situation considered in Ref. [11] is relevant at later times.

Finally, we note that the mean-field description ignores scattering processes; hence, it does not include heating effects and intraband relaxation, which compete with the enhancement of the EI order. However, it can capture important aspects of the short time dynamics [59], and the 
cooperation between collective modes and photoinduced changes in the band structure should survive and play a crucial role in more advanced descriptions. Beyond the mean-field description, we expect that the relaxation dynamics depends on details of the band structure, the spatial profile of the interaction, and the pulse shape. Furthermore, the excitation into additional bands has to be considered in a more realistic description of experiments [11]. These are interesting problems for future investigations.

The authors thank C. Monney, S. Mor, M. Schüler, T. Kaneko, J. Marino, and J. Kuneš for fruitful discussions. Y.M. and D.G. are supported by the Swiss National Science Foundation through NCCR Marvel and Grant No. 200021-165539. P. W. acknowledges support from ERC Consolidator Grant No. 724103.

[1] D. Fausti, R. I. Tobey, N. Dean, S. Kaiser, A. Dienst, M. C. Hoffmann, S. Pyon, T. Takayama, H. Takagi, and A. Cavalleri, Science 331, 189 (2011).

[2] S. Kaiser et al., Phys. Rev. B 89, 184516 (2014).

[3] M. Mitrano et al., Nature (London) 530, 461 (2016).

[4] R. Matsunaga, Y. I. Hamada, K. Makise, Y. Uzawa, H. Terai, Z. Wang, and R. Shimano, Phys. Rev. Lett. 111, 057002 (2013).

[5] R. Matsunaga, N. Tsuji, H. Fujita, A. Sugioka, K. Makise, Y. Uzawa, H. Terai, Z. Wang, H. Aoki, and R. Shimano, Science 345, 1145 (2014).

[6] R. Matsunaga, N. Tsuji, K. Makise, H. Terai, H. Aoki, and R. Shimano, Phys. Rev. B 96, 020505 (2017).

[7] T. Rohwer et al., Nature (London) 471, 490 (2011).

[8] S. Hellmann et al., Nat. Commun. 3, 1069 (2012).

[9] M. Porer, U. Leierseder, J.-M. Ménard, H. Dachraoui, L. Mouchliadis, I. E. Perakis, U. Heinzmann, J. Demsar, K. Rossnagel, and R. Huber, Nat. Mater. 13, 857 (2014).

[10] D. Golež, P. Werner, and M. Eckstein, Phys. Rev. B 94, 035121 (2016).

[11] S. Mor et al., Phys. Rev. Lett. 119, 086401 (2017).

[12] D. Werdehausen et al., arXiv:1611.01053.

[13] D. Jérome, T. M. Rice, and W. Kohn, Phys. Rev. 158, 462 (1967).

[14] W. Kohn, Phys. Rev. Lett. 19, 439 (1967).

[15] B. Halperin and T. Rice, Solid State Phys. 21, 115 (1968).

[16] H. Cercellier et al., Phys. Rev. Lett. 99, 146403 (2007).

[17] C. Monney et al., Phys. Rev. B 79, 045116 (2009).

[18] Y. Wakisaka, T. Sudayama, K. Takubo, T. Mizokawa, M. Arita, H. Namatame, M. Taniguchi, N. Katayama, M. Nohara, and H. Takagi, Phys. Rev. Lett. 103, 026402 (2009).

[19] Y. Wakisaka et al., J. Supercond. Novel Magn. 25, 1231 (2012).

[20] K. Seki et al., Phys. Rev. B 90, 155116 (2014).

[21] Y. F. Lu, H. Kono, T. I. Larkin, A. W. Rost, T. Takayama, A. V. Boris, B. Keimer, and H. Takagi, Nat. Commun. 8, 14408 (2017).

[22] V.-N. Phan, K. W. Becker, and H. Fehske, Phys. Rev. B 81, 205117 (2010).
[23] B. Zenker, D. Ihle, F. X. Bronold, and H. Fehske, Phys. Rev. B 85, 121102 (2012).

[24] T. Kaneko, T. Toriyama, T. Konishi, and Y. Ohta, Phys. Rev. B 87, 035121 (2013).

[25] B. Zenker, H. Fehske, and H. Beck, Phys. Rev. B 90, 195118 (2014).

[26] C. Monney, C. Battaglia, H. Cercellier, P. Aebi, and H. Beck, Phys. Rev. Lett. 106, 106404 (2011).

[27] K. Sugimoto, T. Kaneko, and Y. Ohta, Phys. Rev. B 93, 041105 (2016).

[28] K. Sugimoto and Y. Ohta, Phys. Rev. B 94, 085111 (2016).

[29] H. Matsuura and M. Ogata, J. Phys. Soc. Jpn. 85, 093701 (2016).

[30] L. Balents, Phys. Rev. B 62, 2346 (2000).

[31] J. Kuneš, J. Phys. Condens. Matter 27, 333201 (2015).

[32] J. Nasu, T. Watanabe, M. Naka, and S. Ishihara, Phys. Rev. B 93, 205136 (2016).

[33] See Supplemental Material at http://link.aps.org/ supplemental/10.1103/PhysRevLett.119.247601 for details on the mean-field description, the role of different phonon modes, the dynamical phase transition and the dynamics in the BEC-BCS crossover regime.

[34] P. W. Anderson, Phys. Rev. 112, 1900 (1958).

[35] In Ref. [20], another set of parameters is considered, which turns out to be in the BCS regime at $T=0$. Even there, the light enhancement of the EI phase can be observed; see Supplemental Material.

[36] We evaluate the susceptibility by following $2 \operatorname{Re} \phi(t)$ after an initial perturbation $E(t)=d_{f} \delta(t)$ with small enough $d_{f}$.

[37] I. Kulik, O. Entin-Wohlman, and R. Orbach, J. Low Temp. Phys. 43, 591 (1981).

[38] Y. Murakami, P. Werner, N. Tsuji, and H. Aoki, Phys. Rev. B 93, 094509 (2016).

[39] Y. Murakami, P. Werner, N. Tsuji, and H. Aoki, Phys. Rev. B 94, 115126 (2016).

[40] V. Gurarie, Phys. Rev. Lett. 103, 075301 (2009).

[41] A. Volkov and S. Kogan, Sov. Phys. JETP 38, 1018 (1974).

[42] E. A. Yuzbashyan, O. Tsyplyatyev, and B. L. Altshuler, Phys. Rev. Lett. 96, 097005 (2006).

[43] R. A. Barankov and L. S. Levitov, Phys. Rev. Lett. 96, 230403 (2006).

[44] E. A. Yuzbashyan and M. Dzero, Phys. Rev. Lett. 96, 230404 (2006).

[45] T. Kaneko, B. Zenker, H. Fehske, and Y. Ohta, Phys. Rev. B 92, 115106 (2015).

[46] The modes have been determined by Fourier transformation of $\chi_{11}^{R}(t)$ with a damping $\eta=0.006$.

[47] The gap at each $k[\Delta(k, t)]$ is evaluated by diagonalizing the mean-field Hamiltonian at each time, i.e., $\Delta(k, t)=\left|\mathbf{B}_{k}(t)\right|$. With the present parameters, $\Delta(0,0)=\Delta_{\mathrm{EI}}$.

[48] M. Eckstein, M. Kollar, and P. Werner, Phys. Rev. Lett. 103, 056403 (2009).

[49] M. Schiró and M. Fabrizio, Phys. Rev. Lett. 105, 076401 (2010).

[50] B. Sciolla and G. Biroli, Phys. Rev. Lett. 105, 220401 (2010).

[51] A. Chiocchetta, M. Tavora, A. Gambassi, and A. Mitra, Phys. Rev. B 91, 220302 (2015).

[52] M. Heyl, Phys. Rev. Lett. 113, 205701 (2014).

[53] B. Zunkovic, M. Heyl, M. Knap, and A. Silva, arXiv: 1609.08482 . 
[54] A. Chiocchetta, A. Gambassi, S. Diehl, and J. Marino, Phys. Rev. Lett. 118, 135701 (2017).

[55] A. Lerose et al., arXiv:1706.05062.

[56] J. K. Freericks, H. R. Krishnamurthy, and T. Pruschke, Phys. Rev. Lett. 102, 136401 (2009).
[57] M. Eckstein and M. Kollar, Phys. Rev. B 78, 245113 (2008).

[58] S. Hoshino and P. Werner, Phys. Rev. Lett. 115, 247001 (2015).

[59] N. Tsuji, M. Eckstein, and P. Werner, Phys. Rev. Lett. 110, 136404 (2013). 